\title{
Fast HEVC Intra Prediction Algorithm with Enhanced Intra Mode Grouping Based on Edge Detection in Transform Domain
}

\author{
Miso Park and Jechang Jeong
}

\begin{abstract}
High efficiency video coding (HEVC) is the next generation video coding standard which intended to provide not only substantially improved video quality with the same bit rate but also achieved compression ratio up to $50 \%$ compare to H.264/AVC. To accomplish data compression, HEVC adopted different coding tools such as coding tree unit (CTU), increased number of intra prediction modes, Sample Adaptive Offset (SAO) and so on. In this paper, we deal with intra prediction which has high computational complexity due to the number of prediction modes. To lessen computational load, proposed algorithm suggest two methods. Firstly, proposed algorithm converted each PU into transform domain to classify major directionality. Depend on directionality, classified intra mode group is provided as candidate modes. Secondly, we update intra mode group with certain conditions. Compared to HM 14.0, proposed algorithm provided more than $25 \%$ of encoding time reduction with insignificant coding efficiency loss.
\end{abstract}

Index Terms-Fast intra mode decision, HEVC, intra prediction, transform domain edge detection, video coding.

\section{INTRODUCTION}

Because of needs for high video resolutions and real time broadcasting services, image processing industry required advanced video compression standard compare to H.264/AVC. To overcome difficulty of transmitting and storing high definition video streams, the next generation video coding standard named High Efficiency Video Coding (HEVC) developed [1]. HEVC established by the Joint Collaborative Team on Video Coding (JCT-VC) which is a joint team of ISO/IEC Moving Picture Experts Group (MPEG) and ITU-T Video Coding Experts Group (VCEG). To provide more than $50 \%$ coding efficiency with better visual quality compare to H.264/AVC, significant coding tools adopted, for example, quad-tree based coding unit, Sample Adaptive Offset (SAO), Advanced Motion VectorPrediction (AMVP) and etc. [2]. In previous work of H.264/AVC, only 9 prediction modes were adopted to reduce spatial redundancy for intra prediction. Furthermore, H.264/AVC supports 3 different coding block sizes, $4 \times 4,8 \times 8$, $16 \times 16$, which are insufficient to deal with high resolution sequences [3]-[6]. But in case of HEVC, it supports up to 35

Manuscript received July 12, 2014; revised March 12, 2015. This research was supported by the MSIP (Ministry of Science, ICT \& Future Planning), Korea, under the ITRC (Information Technology Research Center) support program supervised by the NIPA (National IT Industry Promotion Agency) (NIPA-2014-H0301-14-1018).

The authors are with the Department of Electronic and Computer Engineering, Hanyang University, Seoul, Korea (e-mail: misosmile1128@gmail.com,jjeong@hanyang.ac.kr). intra prediction modes and uses various block sizes range from $4 \times 4$ to $64 \times 64$ pixels [7]-[9].

In addition, HEVC includes three types of coding blocks; coding unit (CU), prediction unit (PU), transform unit (TU). Coding Tree Unit (CTU) is a concept of largest coding unit which size is usually set to $64 \times 64$. And CU has a square shape quad-tree structure which size range from $8 \times 8$ to $64 \times 64$. The CU concept allows CTU to be split recursively with four equally sized CU. After splitting CU, PU and TU split itself independently, and their size cannot be larger than the size of $\mathrm{CU}$ [10], [11]. For intra prediction, PU can only split into two types; $\mathrm{N} \times \mathrm{N}$ and $2 \mathrm{~N} \times 2 \mathrm{~N}$. With split PU, HEVC perform 35 intra prediction modes and choose best matching prediction modes to store. Partitioning structure of $\mathrm{CU}$ and $\mathrm{PU}$ is shown in Fig. 1.

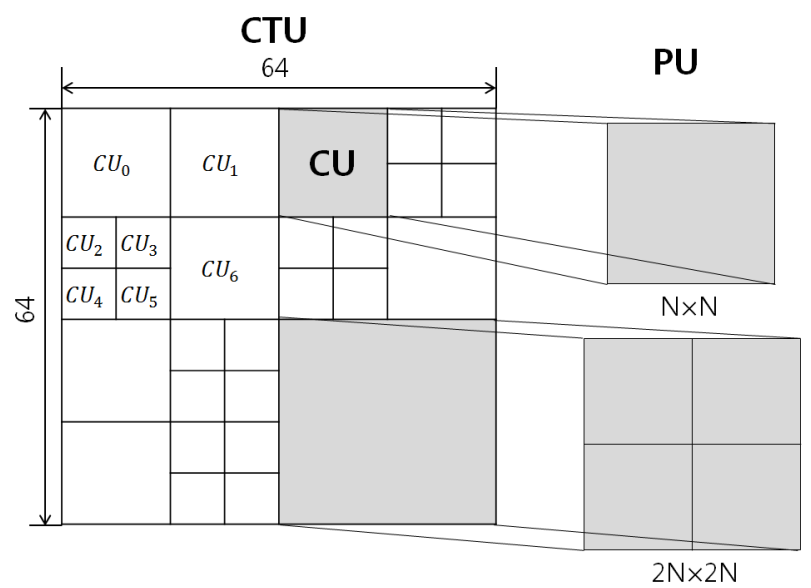

Fig. 1. Partitioning structure of $\mathrm{CU}$ and PU.

Since HEVC operate whole size of coding units and run rate-distortion optimization (RDO) process, another problem called computational complexity occurred. To solve massive amount of operations and bits, especially for intra prediction, a lot of fast intra prediction algorithms were proposed.

In this paper, we proposed fast intra prediction algorithm by analyzing coefficients in DCT domain and grouping intra prediction modes which can eliminate prediction modes with low probability in order to bring less encoding time and computational load. For further improvement of coding efficiency, proposed algorithm update intra mode group by investigating prediction modes of reference blocks. Due to reduced candidates of prediction modes for RDO and rough mode decision (RMD) processes, we confirmed encoding time reduction with negligible visual quality loss.

The remainder of this paper organized as follows. Section II presents overview of intra prediction in HEVC. Section III 
gives details of proposed fast intra prediction algorithm in this paper. Experimental results and conclusions are given in Section IV and Section V, respectively.

\section{OVERVIEW OF INTRA PREDICTION IN HEVC}

Intra prediction is a coding tool which employed to remove spatial redundancies using neighboring pixels within one image. To find exact directions and remove redundancies effectively, HEVC employed up to 35 prediction modes for each PU. Fig. 2 shows 33 angular modes, DC mode, and planar mode for intra prediction [12].

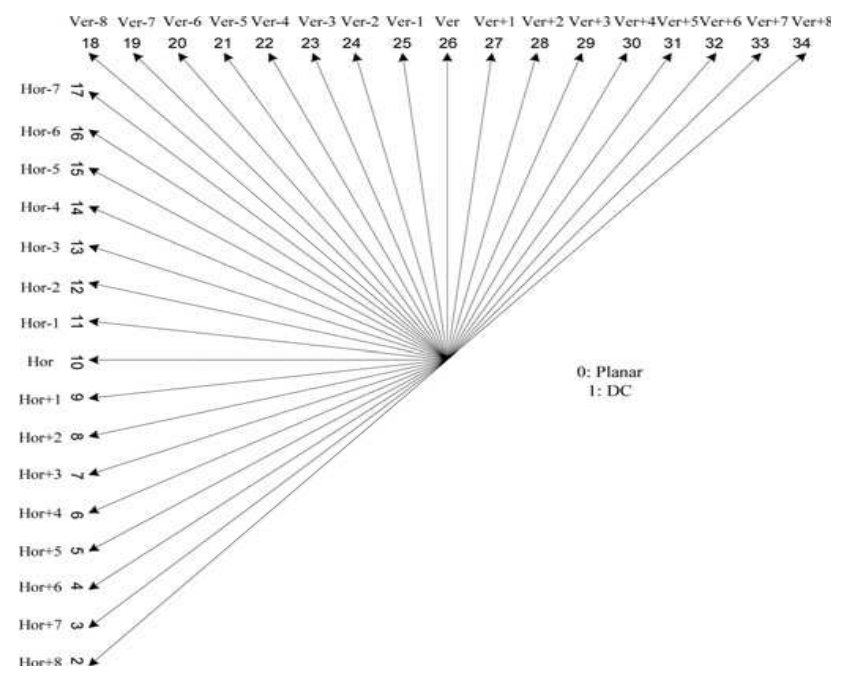

Fig. 2. Intra prediction mode directions.

Basically, for every PU size, all prediction modes should be calculated in the RDO process. However, it will be very time consuming and burden to the encoder. To solve this problem, intra prediction for HEVC adopted RMD process which select first $\mathrm{N}$ candidate modes as optimal modes with sum of absolute Hadamard transformed differences (SATD) instead of discrete cosine transform (DCT) [13]. And optimal modes will be decided according to following equation.

$$
\begin{aligned}
& J_{\text {pred }, S A T D}=S A T D+\lambda_{\text {pred }} \cdot B_{\text {pred }} \\
& S A T D=\left(\sum_{i, j}|\operatorname{DiffT}(i, j)|\right) / 2
\end{aligned}
$$

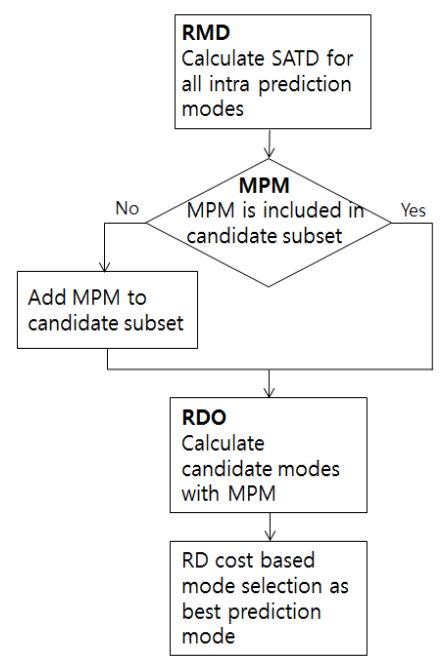

Fig. 3. Flow chart of intra prediction mode selection in HEVC.
Since Hadamard transform perform only with integer add operations, complexity of SATD is much lower than that of DCT. After selecting the subset of optimal modes by RMD process, the most probable mode (MPM) derived from neighboring blocks which will be added to the subset [14]. Finally, RDO process follows to select the mode with minimum rate-distortion cost (RD-cost) as a best prediction mode. Fig. 3 shows flow charts of intra prediction in HEVC.

\section{PROPOSED FAST INTRA PREDICTION ALGORITHM}

Because of Intra prediction in HEVC supports up to 35 directional modes including planar and DC mode for each PU, complexity of encoder for intra prediction take large portion and this should be handle with fast intra prediction algorithms. In this paper, we proposed advanced mode selection with coefficient distribution analysis in DCT domain and grouping intra prediction modes based on characteristics of coefficients for the first step. And for the second step, we detect intra prediction modes of left and upper PU as references to improve coding efficiency or to reduce encoding time which can efficiently lessen complexity of encoder. Details for each step are as follow.

\section{A. Analysis of Intra $4 \times 4$ DCT Coefficient Distribution}

Most of lossy compression like image processing or HEVC, discrete cosine transform adopted since it contains power energy compaction properties. In proposed algorithm, we decide a representative directional mode by performing coefficient distribution analysis in DCT domain by $32 \times 32 \mathrm{PU}$ size for current CTU, and define intra mode group depend on the representative directional mode.

Since complexity of $32 \times 32$ DCT have large portion in the encoder, DCT of $32 \times 32$ PU extract DC coefficients in $168 \times 8$ blocks to create one $4 \times 4$ DC matrix [15]. For the created $4 \times 4$ DC matrix, we analyze coefficients which extracted by $4 \times 4$ DCT to decide its characteristics of directionality. Fig. 4 represents a process of $4 \times 4$ DCT coefficients extraction from $32 \times 32$ PU.

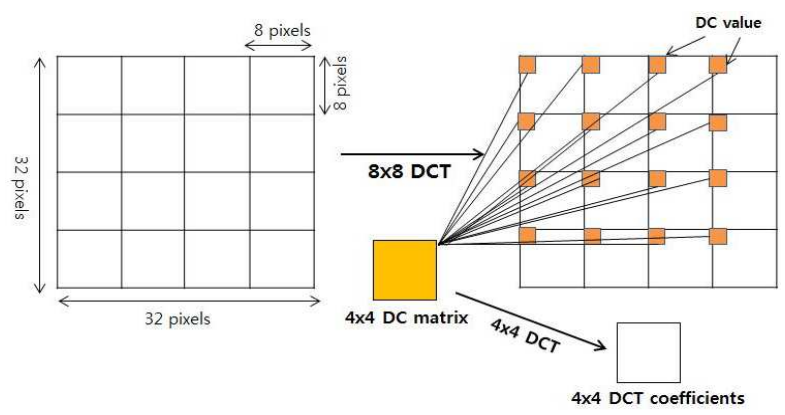

Fig. 4. A process of $4 \times 4$ DCT coefficients extraction.

For more information, characteristics of $4 \times 4$ DCT coefficients are shown in Fig. 5. The coefficients in the upper-left corner of $4 \times 4$ block in the Fig. 5 are DC coefficient which represents the average luminance of the block. The rest of coefficients represent $\mathrm{AC}$ components and each $\mathrm{AC}$ coefficients stand for variations of grey level with certain direction. Although AC coefficients keep information of edge directions, it is difficult to accurately extract information of edge directions in DCT domain [16]. 


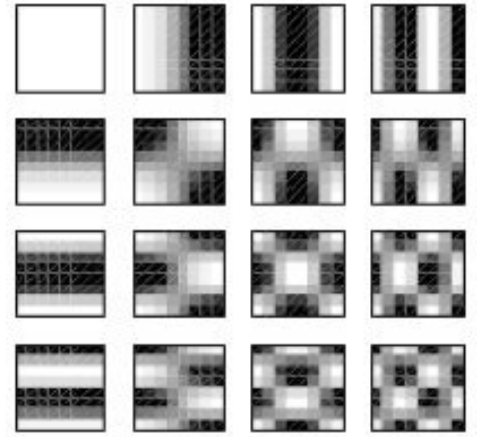

Fig. 5. Analysis of $4 \times 4$ DCT coefficient.

For the improved decision process of prediction directions, we analyzed distribution of $4 \times 4$ DCT coefficients with test blocks shown in Fig. 5. By using test blocks, we estimate intensities of DC, vertical, horizontal, and diagonal directions by the sum of coefficients.

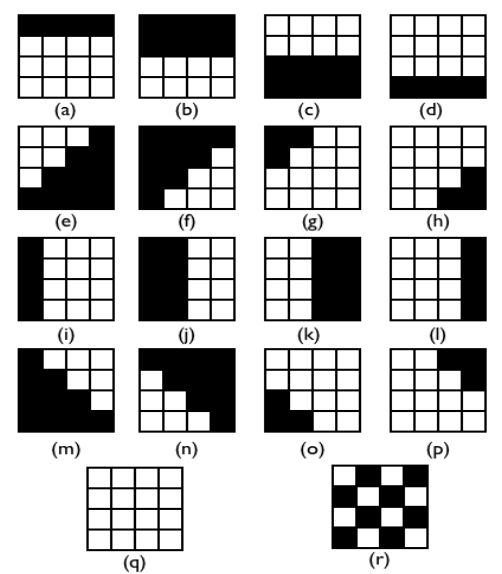

Fig. 6. Test blocks to estimate intensities of coefficients.

And the intensities of each direction are calculated with equation (2). Since proposed algorithm calculated intensities, 18 test blocks which include vertical $\left(0^{\circ}\right)$, diagonal_45 $45^{\circ}$, horizontal $\left(90^{\circ}\right)$, diagonal_135 ${ }^{\circ}$, non-directional, and flat patterns check characteristics of distribution. And from Fig. 6 (e) and (m), we can find the distribution of AC coefficients is symmetric for 45 degree and anti-symmetric for 135 degree, whereas the rate of intensities for each diagonal directions shows equal.

\section{B. Decision of Edge Direction and Intra Mode Grouping}

For the first step to check directionality of $32 \times 32 \mathrm{PU}$, we first determine whether it contains direction or not with the variance of test block. And the variance of blocks in DCT domain is calculated by following equation (3).

$$
\begin{array}{cc}
I_{H}=\sum_{v=0}^{3}|F(0, v)|, \quad(v \neq 0) \\
I_{V}=\sum_{u=0}^{3}|F(u, 0)|, \quad(u \neq 0) \\
I_{D}=\sum_{u=0}^{3}|F(u, u)|, \quad(u \neq 0) \\
I_{D C}=F(0,0) & \\
\sigma^{2}=\left(\sum_{u=0}^{3} \sum_{v=0}^{3} F(u, v)^{2}\right) / N^{2}, & (u, v) \neq(0,0)
\end{array}
$$

According to Parseval theorem, the variance in DCT domain is equal to the variance in spatial domain. To reduce computational complexity, we adopted simplified variance which induced by equation (4) instead of equation (3) which contains squared summation.

$$
\sigma^{2} \cong \sum_{u=0}^{3} \sum_{v=0}^{3}|F(u, v)|, \quad(u, v) \neq(0,0)
$$

If the value of variance for $4 \times 4$ DCT coefficients derived from $32 \times 32 \mathrm{PU}$ is bigger than threshold $\left(\tau_{1}\right)$, that block regarded as high activity region which means $32 \times 32 \mathrm{PU}$ contains edge. And if the condition is not satisfied, it means $32 \times 32$ PU consist of flat region. For the second step, proposed algorithm decides major directionality with the proportion of intensities. Intensities of vertical and horizontal direction are defined as $I_{V}$ and $I_{H}$, respectively. If the ratio of $I_{V}$ to $I_{H}$ is larger than threshold $\left(\tau_{2}\right)$ and smaller than threshold $\left(\tau_{3}\right)$ at the same time, it considered to represent diagonal direction. And, if AC coefficients are symmetric, it supposed to be 45 degree, while it represents 135 degree directions when AC coefficients are anti-symmetric. For the rest of the cases, it considered non-directional when the ratio of $I_{D}$ is dominant. And these process are shown at Fig. 7.

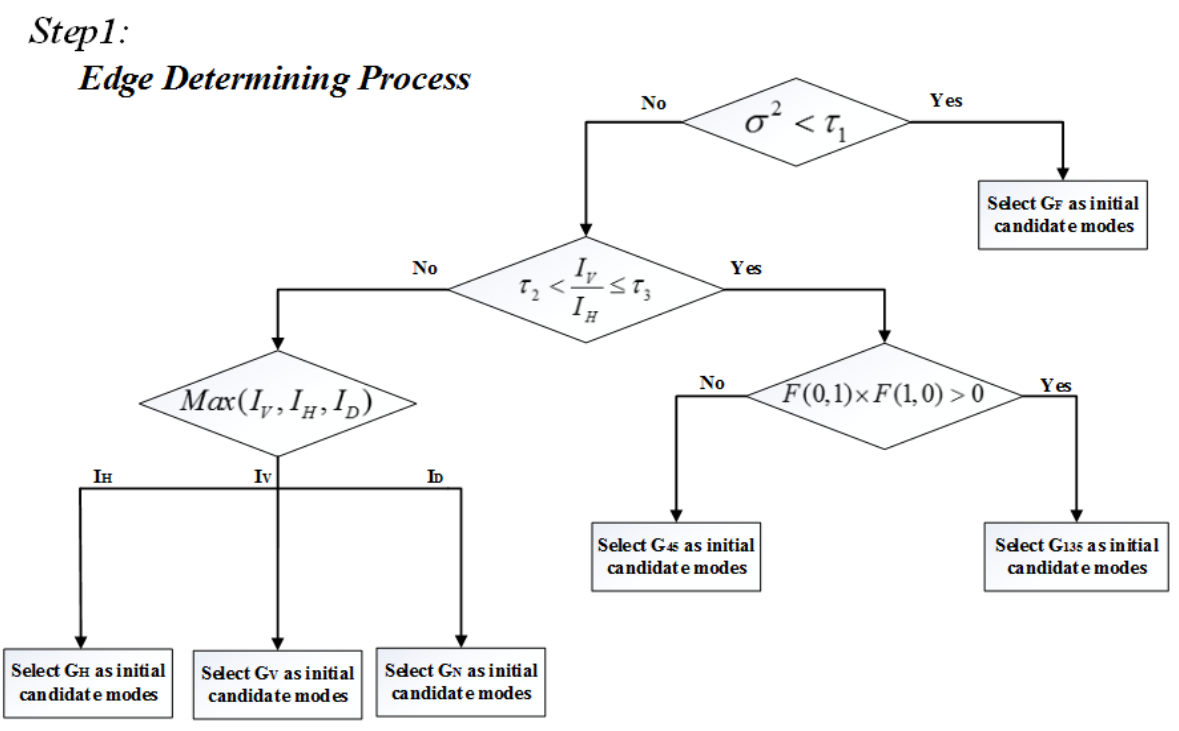




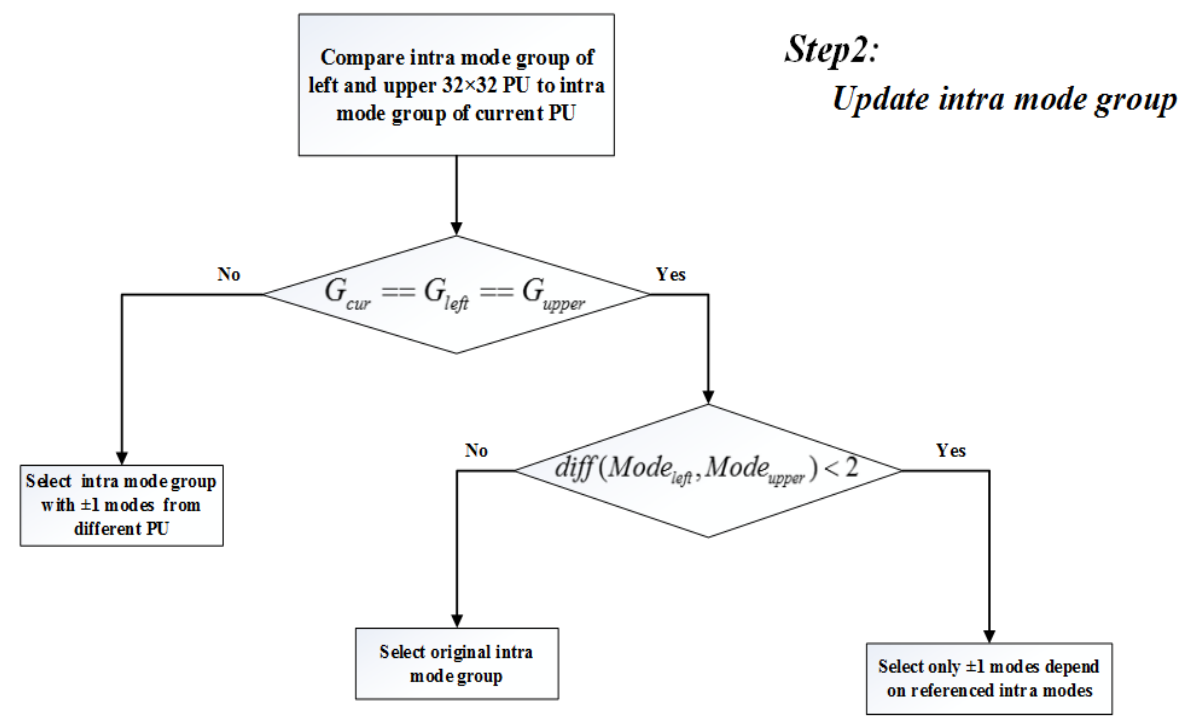

Fig. 7. Flowcharts of the proposed algorithm.

TABLE I: CLASSIFICATION OF INTRA MOde GROUP

\begin{tabular}{cc}
\hline \hline Intra mode group & Prediction mode \\
\hline$G_{H}$ & $6,7,8,9,10,11,12,13,14$ \\
$G_{45}$ & $14,15,16,17,18,19,20,21,22$ \\
$G_{V}$ & $22,23,24,25,26,27,29,30$ \\
$G_{135}$ & $30,31,32,33,34,2,3,4,5,6$ \\
$G_{N}$ & $2,6,10,14,18,22,26,30,34$ \\
$G_{F}$ & DC, planar, 10,26 \\
\hline \hline
\end{tabular}

After deciding major directionality of $32 \times 32$ PU, intra mode grouping based on major directionality will be follow. Depend on a major directionality, we classified prediction modes with six different groups, $G_{H}, G_{45}, G_{V}, G_{135}, G_{N}$, and $G_{F}$ which represent group of horizontal, diagonal $45^{\circ}$, vertical, diagonal_135 ${ }^{\circ}$, non-directional, and flat, respectively. According to classification, intra prediction mode groups are shown in Table I, and edge determining algorithm described in following pseudo code.

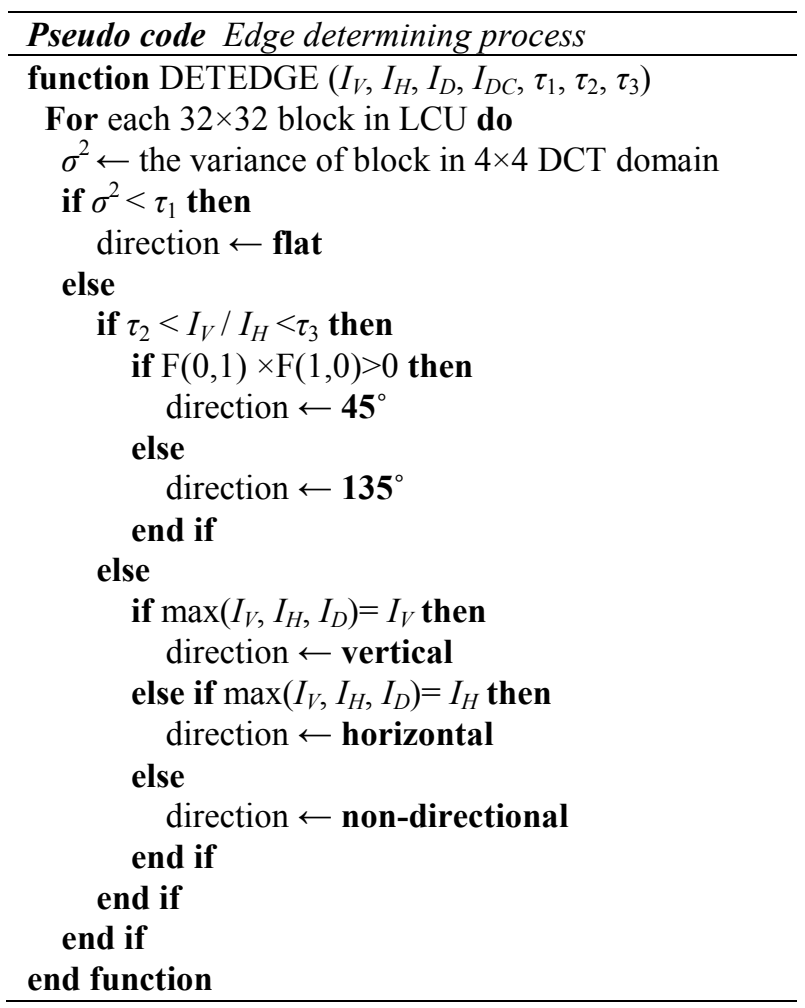

\section{Modification of Intra Mode Candidates}

We defined major directionality for each $32 \times 32 \mathrm{PU}$ in current CTU, and proposed intra mode grouping method depend on major directionality. However, since proposed algorithm use same intra mode group until next $32 \times 32$ PU starts encoding, it might bring coding efficiency loss.

To cover this problem, updating intra mode group algorithm applied. In this step, mode information of left and upper block is used as reference with major directionality. We compare intra mode of left and upper $32 \times 32 \mathrm{PU}$ to intra mode group of current PU. If the left and upper block belong to same intra mode group, proposed algorithm compare intra mode of current PU with that of left and upper PU which has same size. By comparing two modes, and if the difference of the modes is lower than 2, current PU only use \pm 1 modes depend on referenced intra modes. Otherwise, we perform intra prediction for original intra mode group. However, if the difference of the modes are larger than 2 , we assume intra mode group of left and upper $32 \times 32 \mathrm{PU}$ and that of current $32 \times 32$ PU are different. In this case, intra mode group of current PU add \pm 1 modes from different PU, and run intra prediction with updated intra mode group. By adopting modified process for intra prediction, proposed algorithm finally accomplished not only increment of coding efficiency but also reduction of encoding time.

\section{EXPERIMENTAL RESULTS}

\section{A. Test Conditions}

Proposed algorithm implemented in the JCT-VC test model HM 14.0 of HEVC. The test platform is CPU of Intel® core i5-4670 with $3.40 \mathrm{GHz}, 8.00 \mathrm{~GB}$ RAM. To focus on evaluating the performance of the proposed algorithm, only All-Intra (AI) mode with Main profile were used. And, 15 video sequences in five classes (class B to E) were covered with four quantitation parameters, 22, 27, 32, and 37. In order to verify proposed algorithm with the objective quality differences, Bjøntegaard delta bitrates (BDBR) and Bjøntegaard delta peak signal to noise ratio (BDPSNR) calculated as criterion. 


\section{B. Experimental Results}

For the objective comparison, Table II shows experimental results with BDBR [\%], BDPSNR [dB] and time saving (T) (\%). And time saving is defined in equation (5).

$$
T=\frac{1}{4} \sum_{i=1}^{4} \frac{\operatorname{Time}_{H M 14.0}\left(Q P_{i}\right)-\text { Time }_{\text {Proposed }}\left(Q P_{i}\right)}{\operatorname{Time}_{H M 14.0}\left(Q P_{i}\right)} \times 100
$$

From Table II, we can find that our proposed algorithm obtained increment of BDBR about $2.70 \%$ on average with negligible BDPSNR loss. Furthermore, proposed method saved encoding time more than $25 \%$ compare to HM 14.0.

TABLE II: COMPARISON OF PERFORMANCE

\begin{tabular}{|c|c|c|c|c|}
\hline Class & Sequence & Time saving & BDBR & BDPSNR \\
\hline \multirow{4}{*}{$\begin{array}{c}\text { B } \\
{[1920 \times 1080]}\end{array}$} & BQTerrace & 24.06 & 2.99 & -0.17 \\
\hline & Cactus & 31.46 & 3.06 & -0.11 \\
\hline & Kimono & 29.02 & 2.02 & -0.06 \\
\hline & ParkScene & 25.45 & 2.38 & -0.10 \\
\hline \multirow{4}{*}{$\begin{array}{c}\mathrm{C} \\
{[832 \times 480]}\end{array}$} & BasketballDrill & 30.57 & 3.24 & -0.14 \\
\hline & BQMall & 29.01 & 2.06 & -0.12 \\
\hline & PartyScene & 24.41 & 3.24 & -0.25 \\
\hline & RaceHorsesC & 24.38 & 3.23 & -0.20 \\
\hline \multirow{4}{*}{$\frac{\mathrm{D}}{[416 \times 240]}$} & BasketballPass & 24.66 & 2.35 & -0.13 \\
\hline & BlowingBubbles & 26.84 & 3.28 & -0.2 \\
\hline & BQSquare & 28.32 & 2.39 & -0.21 \\
\hline & RaceHorses & 20.91 & 2.11 & -0.14 \\
\hline \multirow{4}{*}{$\begin{array}{c}\mathrm{E} \\
{[1280 \times 720]}\end{array}$} & FourPeople & 17.80 & 2.62 & -0.15 \\
\hline & Johnny & 19.83 & 2.79 & -0.11 \\
\hline & KristenAndSara & 18.43 & 2.73 & -0.14 \\
\hline & Average & 25.01 & 2.70 & -0.15 \\
\hline
\end{tabular}

\section{CONCLUSION}

This paper proposed a fast intra prediction algorithm with two different approaches to lessen computational complexity and let encoding time faster than original HEVC standard. Firstly, proposed algorithm transforms $32 \times 32$ PU into $4 \times 4$ DC matrix to make it easier to define a major directionality. After transforming, $4 \times 4$ DC matrix covered with test blocks and several add operations. Depend on a major directionality, predefined intra mode group applied to RMD and RDO process. Since proposed algorithm use same group until next $32 \times 32$ PU starts encoding, there might be loss of coding efficiency. To handle this problem, updating intra mode group is applied. Through those three steps of algorithm, we obtained time saving for more than $25 \%$ on average with slight visual quality loss. Moreover, our algorithm can be combined with various edge detection methods and fast mode decision for future works.

\section{ACKNOWLEDGMENT}

This research was supported by the MSIP (Ministry of Science, ICT \& Future Planning), Korea, under the project for standardization and research \& development of information communication \& broadcasting. (201400000001132).

\section{REFERENCES}

[1] K. Ugur et al., "High performance, low complexity video coding and the emerging HEVC standard," IEEE Trans. Circuits Syst. Video Technol., vol. 20, no. 12, pp. 1688-1697, December 2010.

[2] G. J. Sullivan, J. R. Ohm, W. J. Han, and T. Wiegand, "Overview of the high video coding (HEVC) standard," IEEE Trans. Circuits Syst. Video Technol., vol. 22, no. 12, pp.1649-1668, December 2012.
[3] T. Wiegand, G. J. Sullivan, G. Bjontegaard, and A. Luthra, "Overview of the H.264/AVC video coding standard," IEEE Trans. Circuits Syst. Video Technol., vol. 13, no. 7, pp. 560-576, July 2003.

[4] S. K. Kwon, A. Punchihewa, D. G. Bailey et al., "Adaptive simplification of prediction modes for H.264 intra-picture coding," IEEE Trans. Broadcast., vol. 58, no. 1, pp. 125-129, March 2012.

[5] C. H. Tseng et al., "Fast sum of absolute transformed difference based $4 \times 4$ intra-mode decision of H.264/AVC video coding standard," IEEE Trans. Circuits Syst., vol. 3, pp. 2128-2131, May 2005.

[6] H. Q. Zeng, K. K. Ma, and C. Cai, "Hierarchical intra mode decision for H.264/AVC," IEEE Trans. Circuits Syst. Video Technol., vol. 20, no. 6, pp. 907-912, June 2010.

[7] WD1: Working draft 1 of high-efficiency video coding, presented at the JCTVC-C403, JCT-VC Meeting, Guangzhou, October 2010.

[8] L. Zhao, L. Zhang, X. Zhao, S. Ma, D. Zhao, and W. Gao, "Further encoder improvement for intra mode decision," presented at the JCT-VC Meeting, Daegu, January 2011.

[9] L.-L. Wang and W.-C. Siu, "Adaptive algorithm for intra prediction with compromised modes skipping and signal processes in HEVC," IEEE Trans. Circuits Syst., vol. 23, no. 10, pp. 1686-1694, 2013.

[10] C.-T. Huang, M. Tikekar, and A. P. Chandrakasan, "Memory-hierarchical and mode-adaptive HEVC intra prediction architecture for quad full HD video decoding," IEEE Trans. Very Large Scale Integr. (VLSI) Syst., vol. 22, no. 7, pp. 1515-1525, 2014.

[11] I.-K. Kim, J. Min, T. Lee, W.-J. Han, and J. H. Park, "Block partitioning structure in the HEVC standard," IEEE Trans. Circuits Syst., vol. 22, no. 12, pp. 1697-1706, December 2012.

[12] L. Shen, Z. Zhang, and Z. Liu, "Effective CU size decision for HEVC intra coding," IEEE Trans. Image Process., vol. 23, no. 10, pp. 4232-4241, October 2014.

[13] S. Cho, and M. Kim, "Fast CU splitting and pruning for suboptimal CU partitioning in HEVC intra coding," IEEE Trans. Circuits Syst. Video Technol., vol. 23, pp. 1555-1564, September 2013.

[14] A. Saxena and F. C. Fernandes, "DCT/DST-based transform coding for intra prediction in image/video coding," IEEE Trans. Image Process., vol. 22, no. 10, pp. 3974-3981, October 2013.

[15] Y. Piao, J. Y. Min, and J. L. Chen, "Encoder improvement of unified intra prediction," presented at the JCTVC-C042, Guangzhou, October 2010.

[16] A. Saxena and F. C. Fernandes, "Mode dependent DCT/DST for intra prediction in block-based image/video coding," in Proc. IEEE Int. Conf. Image Process., September 2011, pp. 1721-1724.

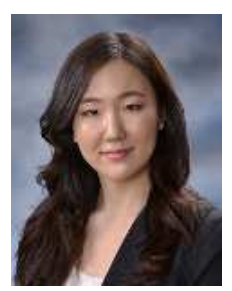

Miso Park received the B.S. degree from the Department of Electronic Engineering, Hanyang University, Korea, in 2013. She is currently a M.S student in the Department of Electronics and Computer Engineering, Hanyang University. Her research interests include motion estimation and compensation algorithms, video coding standards such as HEVC, and image enhancement.

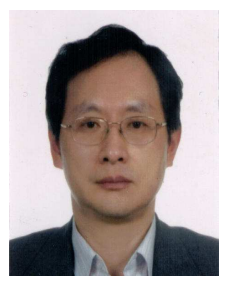

Jechang Jeong received the B.S. degree in electronic engineering from Seoul National University, Korea, in 1980 , the M.S. degree in electrical engineering from the Korea Advanced Institute of Science and Technology in 1982, and the Ph.D. degree in electrical engineering from the University of Michigan, Ann Arbor, MI, USA, in 1990. From 1982 to 1986, he was with the Korean Broadcasting System, where he helped develop teletext systems. From 1990 to 1991, he worked as a postdoctoral research associate at the University of Michigan, Ann Arbor, where he helped to develop various signal-processing algorithms. From 1991 through 1995, he was with the Samsung Electronics Company, Korea, where he was involved in the development of HDTV, digital broadcasting receivers, and other multimedia systems. Since 1995, he has conducted research at Hanyang University, Seoul, Korea. His research interests include digital signal processing, digital communication, and image and audio compression for HDTV and multimedia applications. He has published numerous technical papers. Dr. Jeong received the Scientist of the Month Award in 1998, from the Ministry of Science and Technology of Korea, and was the recipient of the 2007 IEEE Chester Sall Award and 2008 ETRI Journal Paper Award. He was also honored with a government commendation in 1998 from the Ministry of Information and Communication of Korea. 